\title{
Cytogenetic causes for recurrent spontaneous abortions - an experience of 742 couples (1484 cases)
}

\author{
S. Dubey, M. R. Chowdhury, B. Prahlad, V. Kumar, R. Mathur, S. Hamilton, M. Kabra, \\ P. S. N. Menon, I. C. Verma \\ Genetics Unit, Department of Pediatrics, All India Institute of Medical Sciences, Ansari Nagar, New Delhi, India
}

BACKGROUND: First trimester pregnancy loss is a very common complication and a matter of concern for couples planning pregnancy. Balanced chromosomal rearrangements in either parent is an important cause of recurrent pregnancy loss particularly in the first trimester. AIMS: In this study an evaluation of the contribution of chromosomal anomalies in causing repeated spontaneous abortions was made.

METHODS AND MATERIALS: A review of the cytogenetic data in 742 couples (1484 individuals) with recurrent spontaneous abortions who were examined for chromosomal aberrations in the period 1990-2003 is presented. Women who had at least two abortions, or spontaneous abortions preceded or followed by fetal deaths or birth of a malformed child, and patients who had recurrent spontaneous abortions $(>3$ ) with normal live issue/s were studied.

RESULTS: Chromosomal rearrangements were found in 31 individuals (2\%). These abnormalities included 22 $(2.9 \%)$ structural aberrations, 9 (1.2\%) numerical anomalies. In addition to these abnormalities, 21 (3.2\%) chromosomal variants were also found.

CONCLUSION: Chromosomal analysis is an important etiological investigation in couples with repeated spontaneous abortions as it helps in genetic counseling and deciding about further reproductive options.

Key words: Chromosomal abnormalities; reciprocal translocation; recurrent spontaneous abortion; Robertsonian translocation.

Early pregnancy loss in the first trimester is the most common complication affecting at least $15-20 \%$ of the clinically recognized pregnancies. ${ }^{[1]}$ Recurrent spontaneous abortion (RSA) is historically defined as three or more consecutive pregnancy losses before 2022 weeks of gestation. ${ }^{[2]}$ However, some investigators feel that even two spontaneous losses constitute recurrent miscarriage and deserve evaluation. In a patient with a history of two miscarriages, the subsequent risk of pregnancy loss rises to about $25 \%$, whereas three abortions raise the risk of a fourth miscarriage to $33 \%$. To date, however there is no clearcut threshold for the number of spontaneous abortions that unequivocally warrants evaluation. It is not unusual for perfectly healthy couples to experience three consecutive spontaneous pregnancy losses, each for a different reason and it has been seen that more than half of recurrent abortions are due to nonrecurrent causes. ${ }^{[3]}$ Determining the cause of RSA can be extremely difficult. Losses during the first trimester usually are due to fetal genetic defects. The causes of RSA can be often related to factors associated with implantation, genetics, autoimmunity, endocrine abnormalities, infection, alloimmunity and anatomic uterine defects. The analysis of aneuploidies, translocations and other gross structural aberrations of the chromosomes have greatly helped to determine the etiology in majority of cases of RSA. Pregnancies lost in late gestations also have a high rate of chromosomal abnormalities, roughly $30 \%$ in the second trimester and $5 \%$ in the third trimester ${ }^{[4]}$ In the present article we have tried to analyze the cytogenetic causes in the couples with spontaneous abortions. 


\section{Methods and materials}

In the present study 742 couples (1484 individuals) with not less than two spontaneous abortions were analyzed for the chromosomal abnormalities from the year 1990 to 2003 in tertiary care hospital. These couples were categorized as (1) couples only with repeated spontaneous abortions (RSA), (2) couples with RSA preceded by stillbirth (SB) or malformed child (MC), and (3) couples with RSA and normal live issue/ $s(\mathrm{NC})$. All the couples were in the age group ranging from 19 to 39 years and number of abortions ranged from 2 to 9. Consent was taken from each couple informing them about the purpose of the study and was counseled accordingly.

Peripheral blood $(2 \mathrm{ml})$ was collected in heparin vacutainers (Becton Dickinson, USA). For every subject whole blood $(0.5 \mathrm{ml})$ cultures was set up in $5 \mathrm{ml}$ RPMI 1640 media (GIBCO BRL, USA) containing 15\% fetal calf serum (Biological Industries, $\mathrm{KBH}$, Israel), antibiotic mixture and phytohemagglutinin P (DIFCO Lab, USA) for $72 \mathrm{~h} \cdot{ }^{[5]}$ Giemsa trypsin banding (GTG) of metaphase chromosomes was performed using standard methodology ${ }^{[6]}$ In each case minimum of 25 complete metaphase plates were scored and karyotypes prepared. A minimum of 50 metaphases were further analyzed for confirmation of mosaicism.

\section{Results}

Among 742 couples (1484 cases) studied, chromosomal abnormalities were found in 31 (2\%) subjects including 24 females and seven males. Among 31 subjects, 22 (71\%) showed structural aberrations, and $9(29 \%)$ carried numerical abnormalities. In addition, $21(1.4 \%)$ individuals were found to have chromosomal variants. Among structural abnormalities that formed the largest group of chromosomal anomalies, reciprocal translocations were seen in 15 cases $(68.2 \%)$, which frequently involved chromosomes $6,8,11$, and 18. Robertsonian translocations were found only in four cases (18.1\%) involving chromosome 22;22, 21;22, and 13;14. Two $(9 \%)$ subjects showed deletions, one each in chromosome 3 (pter $\rightarrow$ q 25) and 10 (pter $\rightarrow$ q22). The deleted portions of these chromosomes were present in all the metaphases appearing as marker. Since both these subjects were clinically normal, it was assumed that there was no loss of chromatin following deletions and these markers were actually the deleted part of the chromosomes which otherwise was quite evident from their banding pattern. One case of multiple translocation involving chromosomes 6,12 , and 13 was also reported in a woman [Table 1]. Inversion involving chromosome 4 was observed only in one (4\%) subject. Numerical anomalies were found in 9 (17.3\%) subjects out of these, $3(33.3 \%)$ subjects were mosaics with monosomy $45, X$ along with normal cell line, 3 (33.3\%) subjects had 46, XXX karyotype with normal cell line whereas 46, XXY karyotype was observed only in one $(13.2 \%)$ subject. Three cell lines showing 47, XXX,

\begin{tabular}{|c|c|c|c|}
\hline S. No. & Reciprocal translocation & Age & Sex \\
\hline 1 & 46,XX t(1;5) (p33:q35) & 30 & $\mathrm{~F}$ \\
\hline 2 & $46, X X$ t(2;11) (q21:q24) & 22 & $\mathrm{~F}$ \\
\hline 3 & $46, X X \mathrm{t}(3 ; 11)$ (q26:11pter) & 28 & $\mathrm{~F}$ \\
\hline 4 & $46, X X t(3 ; 17)(p 22: p 13)$ & 26 & $\mathrm{~F}$ \\
\hline 5 & $46, X X t(6 ; X)(q 32: q 22)$ & 27 & $\mathrm{~F}$ \\
\hline \multirow[t]{2}{*}{6} & $46, X X \mathrm{t}(6 ; 12 ; 13)$ & & \\
\hline & (q22:q21:q23:q12) & 25 & $\mathrm{~F}$ \\
\hline 7 & $46, X X \mathrm{t}(6 ; 18)(q 27: q 21)$ & 22 & $\mathrm{~F}$ \\
\hline 8 & $46, \mathrm{XY} t(7 ; 8)(q 11: p 11)$ & 25 & M \\
\hline 9 & $46, X Y$ t $(7 ; 13)$ (pter:q13) & 32 & M \\
\hline 10 & $46, X X t(8 ; 15)(q 12: p 11)$ & 35 & $\mathrm{~F}$ \\
\hline 11 & 46,XY t(8;18) (q23:p23) & 30 & M \\
\hline 12 & 46,XX t(8;19) (p22:p13.3) & 35 & $\mathrm{~F}$ \\
\hline 13 & $46, X X \mathrm{t}(11 ; 12)(p 15: q 22)$ & 27 & $\mathrm{~F}$ \\
\hline 14 & 46,XX t(15; 8) (p13:q22) & 26 & $\mathrm{~F}$ \\
\hline 15 & $46, X X$ t(16;X) (q28:q24) & 30 & $\mathrm{~F}$ \\
\hline
\end{tabular}

\begin{tabular}{llll}
\hline \multicolumn{2}{l}{ Robertsonian translocation } \\
\hline 1 & $\mathrm{t}(13 ; 14)$ & 22 & $\mathrm{~F}$ \\
2 & $46, \mathrm{XX} \mathrm{t}(13 ; 14)$ & 31 & $\mathrm{~F}$ \\
3 & $46, \mathrm{XXt}(21 ; 22)$ & 30 & $\mathrm{~F}$ \\
4 & $46, \mathrm{XXt}(22 ; 22)$ & 25 & $\mathrm{~F}$ \\
\hline
\end{tabular}

Deletion + marker

$146, X Y d e l(10)($ pter $\rightarrow$ q 22) + marker $30 \mathrm{M}$

24 46,XYdel (3) (pter $\rightarrow$ q25) + marker $36 \mathrm{M0}$

Inversion

$146, X Y \operatorname{inv}(4)(p 15: q 13) \quad 22 \quad M$ 
$45, X$, and $46, X X$ were observed in two $(22.2 \%)$ subjects [Table 2].

Apart from these major chromosomal abnormalities, chromosomal variants were found in 21 cases, which included those with particularly large paracentromeric constitutive heterochromatic blocks, large 1qh and 9qh, inversion of chromosome 9 and variations in $Y$ chromosome [Table 3]. Three cases having fragile sites involving 2q, 9q, 1p, and 16q were also observed.

[Table 4] shows the correlation of the patients with chromosomal abnormalities according to their obstetric history. Chromosomal anomalies were found to be $(4.1 \%)$ in couples with only RSAs. This frequency decreased with the number of abortions per couple. The maximum frequency was observed in cases with 2-5 abortions. In couples with history of SB or MC along

\section{Table 2: Numerical chromosomal abnormalities}

\begin{tabular}{llccc}
\hline S. No Numerical & $\%$ age & Age & Sex \\
\hline & & $42: 5$ & & \\
2 & $47, \mathrm{XXX}: 46, \mathrm{XX}$ & 8 & 35 & $\mathrm{~F}$ \\
& & 5 & 36 & $\mathrm{M}$ \\
& & $45: 5$ & & \\
3 & $47, \mathrm{XXX}: 46, \mathrm{XX}: 4$ & $26: 52:$ & & \\
& $5, \mathrm{XO}$ & 22 & 24 & $\mathrm{~F}$ \\
& & & & \\
4 & $47, \mathrm{XXX}: 46, \mathrm{XX}$ & $25: 7$ & & \\
5 & $47, \mathrm{XXX}: 46, \mathrm{XX}: 4$ & $20: 56:$ & 30 & $\mathrm{~F}$ \\
& $5, \mathrm{XO}$ & 24 & & \\
& & $94:$ & & \\
6 & $46, \mathrm{XX}: 45, \mathrm{XO}$ & 6 & 27 & $\mathrm{~F}$ \\
& & $80:$ & & \\
7 & $46, \mathrm{XX}: 45, \mathrm{XO}$ & 20 & 30 & $\mathrm{~F}$ \\
& & $72:$ & & \\
8 & $46, \mathrm{XX}: 45, \mathrm{XO}$ & 28 & 28 & $\mathrm{~F}$ \\
& & $62:$ & & \\
\hline & $47, \mathrm{XXX}: 46, \mathrm{XX}$ & 38 & 25 & $\mathrm{~F}$ \\
\hline
\end{tabular}

Table 3: Polymorphic chromosomal variants

\begin{tabular}{llcc}
\hline S. No. & Variants & Number of cases & Percentage \\
\hline 1 & $9 q h_{+}$ & 4 & 19.04 \\
2 & Pericentric inv (9) & 3 & 14.28 \\
3 & $22 p+$ & 3 & 14.28 \\
4 & Fragile sites & 3 & 14.28 \\
5 & $16 q h_{+}$ & 2 & 9.52 \\
6 & $15 p+$ & 2 & 9.52 \\
7 & $\mathrm{Yq}_{+}$ & 2 & 9.52 \\
8 & $\mathrm{Yq}_{-}$ & 1 & 4.76 \\
9 & $1 \mathrm{qh}+$ & 1 & 4.76 \\
\hline
\end{tabular}

Table 4: Major chromosomal anomalies according to obstetric history

\begin{tabular}{lccc}
\hline Indications & Number of couples & $\begin{array}{c}\text { Major } \\
\text { chromosomal } \\
\text { anomalies }\end{array}$ & Percentage (\%) \\
\hline $\mathrm{RSA}^{*}$ & 480 & 20 & 4.1 \\
$\mathrm{RSA}$ * & 190 & 7 & 3.6 \\
$+\mathrm{MC} \dagger+\mathrm{SB} \ddagger$ & & \\
$\mathrm{RSA}$ * & & 5.5 \\
$+\mathrm{NC} \S$ & 72 & \\
${ }^{*}$ Repeated spontaneous abortions. \\
† Malformed child. \\
‡ Stillbirth. \\
§ Normal child.
\end{tabular}

with RSA, the frequency of chromosomal aberrations observed was $3.6 \%$, while in couples with RSA and NC, this frequency was the highest at $5.5 \%$.

\section{Discussion}

The evaluation of patients with a history of repeated spontaneous abortions requires careful consideration of potential genetic, anatomic, endocrine, infectious, and immunologic factors. Assigning proper etiological role to each of these contributing factors is often unclear, however the specific information about the cytogenetic makeup of the couples and if possible of the abortus, still remains a primary focus during evaluation of such cases. In this study, the incidence of chromosomal abnormalities among the couples with RSA was $4 \%$ (2\% of individuals), and chromosomal variants were detected in $1.4 \%$ individuals. The frequency reported in literature varies from 2.9 to $5 \%{ }^{[7-9]}$ except few studies, ${ }^{[10],[11]}$ in which higher frequencies have been reported but the number of subjects studied were less. The incidence of chromosomal abnormality in couples with recurrent abortions reported in one cumulative study was $2.86 \%$ on an average, ${ }^{[12]}$ which is quite less than that of the present study.

In this study, 31 cases ( 24 women and seven men) had chromosomal abnormalities, and the female: male ratio is $2.1: 1$. The predominance of females appears to be due to the fact that chromosomal abnormalities that are compatible with fertility in females may be associated with sterility in males. ${ }^{[13]}$

The mean maternal age of subjects carrying chromosomal anomalies was 30.2 years. There was no 
positive correlation of advanced maternal age with the number of abortions observed in these subjects indicating that the chromosomal abnormalities could arise because of some reasons other than advanced maternal age. According to this data about $78.3 \%$ of the abortions were in the first trimester, $20 \%$ in the second and $1.7 \%$ in the third trimester showing a positive correlation between the number of abortions and the gestational age. There was no increase in the rate of chromosomal abnormalities relative to the number of abortions in this study. This is in consonance with earlier reports. ${ }^{[14]}$ High frequency $(5.5 \%)$ of chromosome rearrangements in couples with RSA and NC [Table 4] was also observed and this finding is similar to previous reports. ${ }^{[14-16]}$

Robertsonian translocations were less frequent with a frequency of $12.9 \%$. Two cases with $t(13 q ; 14 q)$ and one each with $\mathrm{t}(22 \mathrm{q} ; 22 \mathrm{q})$ and $\mathrm{t}(21 \mathrm{q} ; 22 \mathrm{q})$ were observed. Studies indicate that when the Robertsonian translocation is maternal, there is greater risk that the fetus will exhibit an unbalanced phenotype ${ }^{[17]}$ Inversions were very rare. Only one case was found involving pericentric region of chromosome 4 . The risk of pregnancy loss with a chromosome inversion is not known. It has been estimated that the risk of miscarriage in couples with reciprocal translocations is approximately $25-50 \%$ whereas with Robertsonian translocation it is approximately $25 \% .{ }^{[4]}$ Deletions were observed in two cases but there was no loss of deleted fragment as it was retained in all the metaphases analyzed. It is thus assumed that gain or loss of this fragment during gametogenesis could have led to the chromosomal imbalance in the fetus resulting in spontaneouse abortion. But since the abortus material was not available for analysis, the exact cause of fetal loss could not be confirmed. Numerical aberrations were found in nine cases, which included mosaics with two or three cell lines. Turner mosaics were the most frequent (55.6\%) followed by mosaicism of polysomy of sex chromosomes (44.4\%).

In the present study a number of minor polymorphic chromosomal variants were observed [Table 3]. In four cases pericentric inversion of chromosome 9 was identified but in most of the studies pericentric inversion of chromosome 9 has been observed in cases without any history of RSA. ${ }^{[18]}$ In two cases the same inversion was also observed in their live issues with facial dysmorphism and other malformations. This indicates the possibility of inversion nine to have a role in the etiology of RSA. But more data and molecular genetic studies are needed to confirm this possibility. Other variants like quantitative (1qh, 9qh, 16qh, and Yqh) and qualitative (15p and 22p) heterochromatic polymorphisms, large satellites and fragments, have been implicated in mitotic instability and a tendency towards an increased risk for aneuploidy. ${ }^{[19]}$

Ideally chromosomal studies should be done on the abortus material to know the contributory cause for that abortion. But since most of the abortions were carried out in the native places, where the patient belonged to, it was not possible to collect the abortus samples. Prenatal diagnosis was carried out in further pregnancies in three couples with balanced reciprocal translocations. Fetal karyotype in a couple with father having 46, XY $\mathrm{t}(7 ; 8)$ and in another case mother having $45, \mathrm{XX} t(21 ; 22)$ was done. In both the cases the fetus was found to be a carrier of the same translocation. Therefore the pregnancies were continued and on follow up no phenotypic abnormality was detected. In the third case, the couple had eight abortions with the mother having multiple translocations $46, X X, t(6 ; 12 ; 13)$. Although no cytogenetic study was carried on the abortuses of this subject, it was reasonable to associate her spontaneous abortions due to unbalanced karyotypes in the fetus. ${ }^{[20]}$ The proband conceived the ninth time after this evaluation. The couple did not give consent for any prenatal testing in view of bad obstetric history. She delivered a full-term normal male baby without any malformations. The karyotype of the baby was done for balanced rearrangement and the baby was found to have 46XY karyotype.

A collaborative study involving 71 European prenatal diagnosis centers found a rate of $3.4 \%$ unbalanced fetal karyotypes in couples in which a parent had a balanced structural rearrangement. ${ }^{[17]}$ Therefore all the couples with balanced translocations should be strongly advised to monitor their future pregnancies by prenatal diagnosis to exclude the possibility of a chromosomally unbalanced zygote. Cytogenetic studies give considerable information about the genetic make up leading to RSA and still remain an important tool. In the coming years, newer advanced techniques will help to increase our 
understanding of RSA resulting in new and expedient diagnosis and potential treatment.

\section{References}

1. Boue A, Boue J, Gropp A. Cytogenetics in pregnancy wastage. In: Haris $\mathrm{H}$, Hirschtorn $\mathrm{K}$, Eds. Advances in human genetics. New York: Plenum Press; 1985. p. 157.

2. Stirrat GM. Recurrent miscarriage. Lancet 1990;336:6735.

3. Coulam CB. Epidemiology of recurrent spontaneous abortion. Am J Reprod Immunol 1991;26:23-7.

4. Lee RM, Silver RM. Recurrent pregnancy loss: summary and clinical recommendations. Sem Reprod Med 2000;18:433-40.

5. Moorhead PS, Norvell PC, Mellman WJ, Battips DM, Hungerford DA. Chromosome preparations of leukocytes cultured from human peripheral blood. Exp Cell Res 1960;202:613-6.

6. Seabright M. A rapid banding technique for human chromosomes. Lancet 1971;2:971-2.

7. Campana M, Serra A, Keri G. Role of chromosome aberrations in recurrent abortion: a study of 269 balanced translocations. Am J Med Genet 1986;24:341-65.

8. Tharapel AT, Tharapel SA, Bannerman RM. Recurrent pregnancy losses and prenatal chromosome abnormalities a review. Br J Obstet Gynecol 1985;92:899914.

9. Braekeleer MD, Dao TN. Cytogenetic studies in couples experiencing repeated pregnancy losses. Hum Reprod 1990;5:519-28.

10. Nordenson I. Increased frequencies of chromosomal abnormalities in families with a history of fetal wastage. Clin Genet 1981;19:168-73.

11. Gupta NJ, Chakravarty BN. Early pregnancy wastage with special reference on chromosomal anomalies. J Indian Med Assoc 1995;93:71-4.

12. Kalana VL, Satynarayana M, Sunil Kumar P, Giri Kumar $\mathrm{CH}$. Chromosomal aberrations in recurrent aborters. Bionature 2004;24:79-84

13. Marmor D, Taillemite JL Van den Akker J, Portnoi MF, le Porrier N, Joye N, et al. Semen analysis on subfertile balanced-translocation carriers. Fertil Steril 1980;34:496502.

14. Portnoi MF, Joye N, Akker JV, Morlier G, Taillemite JL. Karyotypes of 1142 couples with recurrent abortion. Obstet Gynaecol 1988;72:31-4.

15. Husslein $P$, Huber J, Wagenbichler $P$, Schnedl W. Chromosome abnormalities in 150 couples with multiple spontaneous abortions. Fertil Steril 1982;37:379-83.

16. Schwartz S, Palmer CG. Chromosome findings in 164 couples with repeated spontaneous abortions, with special consideration to prior reproductive history. Hum Genet 1983;63:28-34.

17. Boue A, Gallano P. A collaborative study of the segregation of inherited chromosome structural arrangements in 1356 prenatal diagnosis. Prenat Diagn 1984;4:45-67.

18. Mattei MG, Mattei JF, Guichaoua M, Giraud F. Partial inversion of the secondary constriction of chromosome 9. Does it exist? Hum Genet 1981;59:310-6.

19. Ward KJ. Genetic factors in recurrent pregnancy loss. Sem Reprod Med 2000;18:425-32.

20. Dubey S, Mathur R, Kabra M, Menon PS. Detection of complex chromosomal rearrangements in a woman with repeated spontaneous abortions. Acta Obstet Gynecol Scand 2001;80:468-9. 